\title{
Animal Models of Episodic Ataxia Type 1 (EA1)
}

\section{Maria Cristina D'Adamo ${ }^{1,2}$, Giuseppe Di Giovanni ${ }^{2,3}$, Mauro Pessia1, 2}

${ }^{1}$ Department of Experimental Medicine, Section of Physiology and Biochemistry, University of Perugia, Perugia, Italy; ${ }^{2}$ Euro-Mediterranean Institute of Science and Technology, IEMEST, Palermo, Italy; ${ }^{3}$ Department of Physiology \& Biochemistry, University of Malta, Msida, Malta

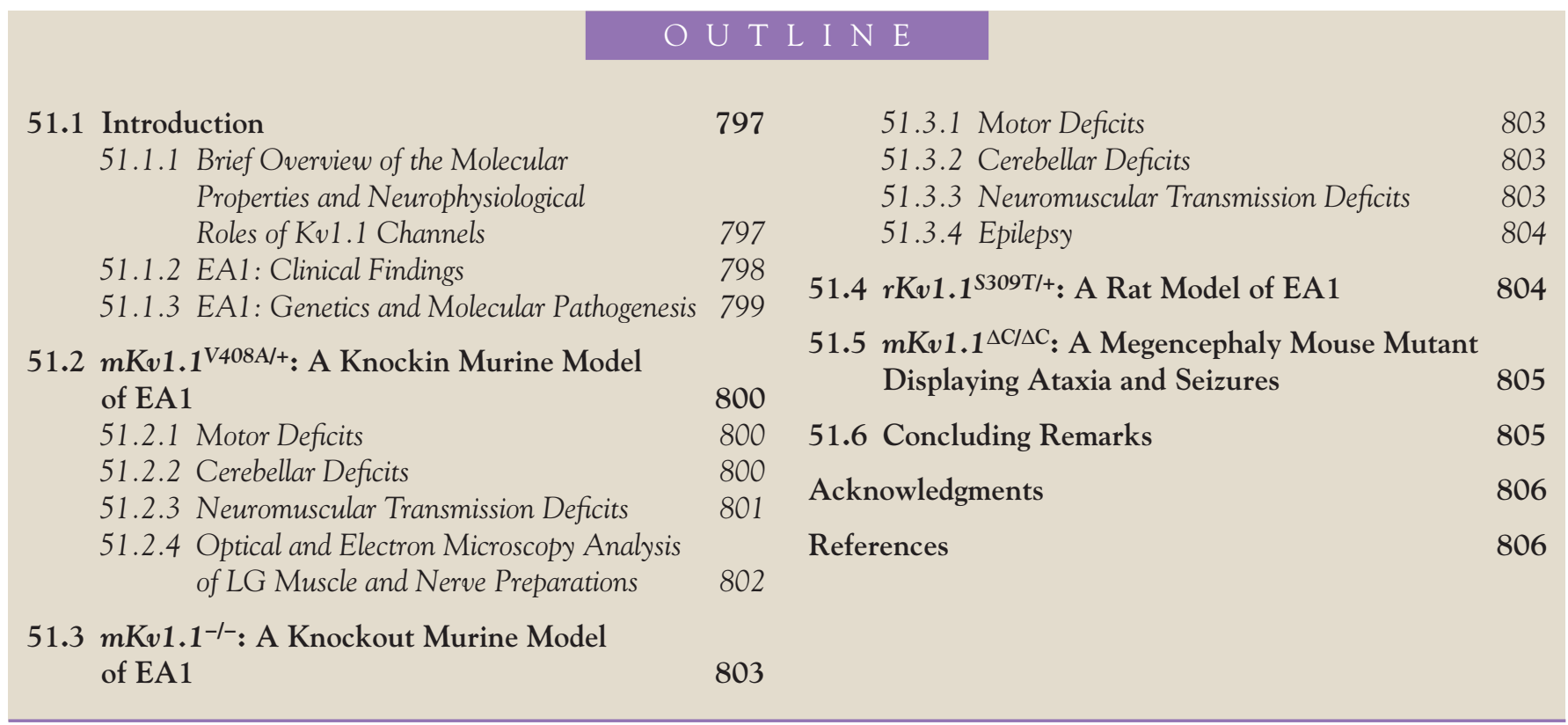

\subsection{INTRODUCTION}

\subsubsection{Brief Overview of the Molecular Properties and Neurophysiological Roles of Kv1.1 Channels}

Full understanding of how mutations in $\mathrm{K}^{+}$channels give rise to distinct human and animal phenotypes requires at least a basic knowledge of their molecular features, expression patterns, and physiological roles. Thus, a brief overview of these topics is included here for the benefit of the readers. Voltage-gated $\mathrm{K}^{+}$channels $(\mathrm{Kv})$ are generally closed at the resting membrane potential of nerve cells, which is approximately $-70 \mathrm{mV}$ (Figure 51.1). Depolarizing steps trigger the opening of
$\mathrm{Kv}$ channels, whereas membrane repolarization closes these channels. At a single channel level, membrane depolarizations elicit channel opening and closing (a process named gating) and are visible as upward and downward deflection of the current traces, respectively (Pessia, 2004).

$\mathrm{K}^{+}$channels are encoded by more than 70 genes, and therefore they are the largest group of ion channels, found in virtually every cell of the human body. The first $\mathrm{Kv}$ channel was cloned from the Shaker mutant of Drosophila melanogaster in 1987 (Tempel et al., 1987). This fruit fly displays a shaking phenotype induced by ether, as the relevant $\mathrm{K}^{+}$channel gene is mutated. Therefore, it may be considered as an animal model of a neuromuscular channelopathy (CP) (Jan et al., 1977; Tanouye and Ferrus, 1985). 


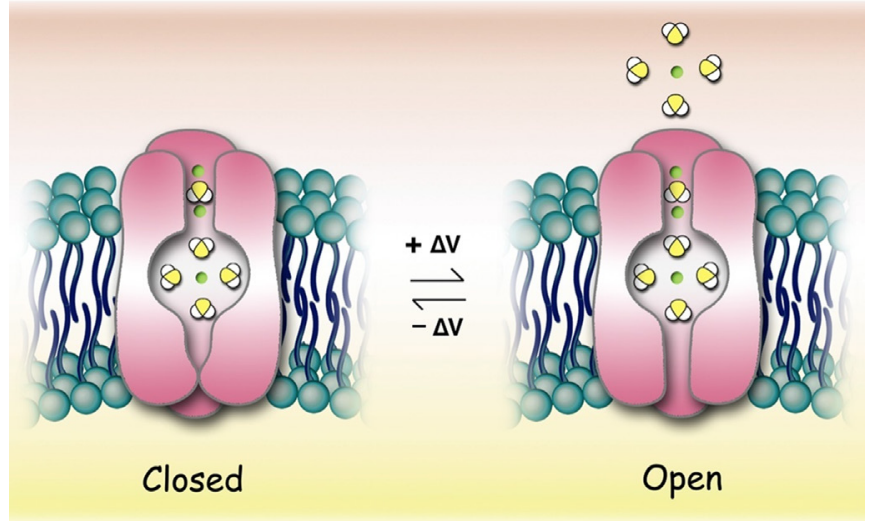

FIGURE 51.1 Illustration recapitulating the main structural changes linked to voltage-gated $\mathrm{K}^{+}$channel gating. The scheme on the left shows a potassium channel in the closed state, with the intracellular gates shut. Membrane depolarization opens the intracellular gates (right), and the $\mathrm{K}^{+}$residing in the cavity sheds its hydration water upon entering the selectivity filter. The $\mathrm{K}^{+}$ion in front is then forced to move outward and gets rehydrated by extracellular water. This mechanism of $\mathrm{K}^{+}$permeation through the pore is very efficient and energetically favorable. The subunit in front has been removed for clarity.

The human ortholog of the Shaker $\mathrm{K}^{+}$channel is encoded by KCNA1 (Kv1.1). Since the first cloning, several other genes encoding for $\mathrm{Kv}$ channels have been identified from many different species. Based on sequence relatedness, Kv channels have been classified in subfamilies using the abbreviation Kvx.y (Gutman and Chandy, 1993). According to this standardized nomenclature, Shaker-related channels have been classified in the subfamily $\mathrm{Kv1}$, and each member is numbered Kv1.1 through Kv1.8 (Figure 51.2). The predicted 496 amino acids of the Kv1.1 protein contain six transmembrane segments (TM) with the $\mathrm{N}$ - and C-termini residing inside the cell (Figure 51.3). TM5, TM6, and the loop linking them contribute to the ion-conducting pore. The glycine-tyrosine-glycine (GYC) residues, residing within such a loop, control the $\mathrm{K}^{+}$selectivity of the channel. The TM4 segment of each Kvx.y subunit is composed of regularly spaced, positively charged arginines and lysines and comprises the main voltage-sensor region that opens the channel by undergoing a conformational rearrangement upon membrane depolarization. Also, the entire crystal structure of a $\mathrm{Kv}$ channel has been provided, confirming that this channel is composed of four homologous pore-forming subunits (Jiang et al., 2003a,b). The description of the structures of the membrane-delimited $\mathrm{Kv}$ channel domain, T1 domain, and $\beta$ subunit allowed the elucidation of many biophysical mechanisms controlling channel function.

$\mathrm{K}^{+}$channels are also the most diverse class of ion channels because they may exist as homomers whenever four identical $\alpha$-subunits are assembled. However, different types of $\alpha$-subunits may heteropolymerize to form channels with properties that are different from the

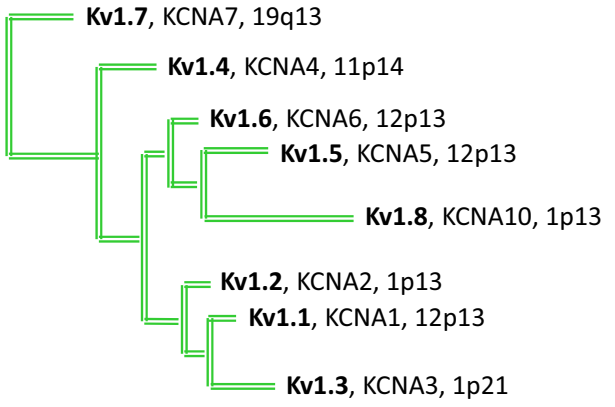

FIGURE 51.2 Phylogenetic tree of the Kv1 family.

parental homomeric channels (Ruppersberg et al., 1990; Isacoff et al., 1990). This phenomenon greatly enhances the diversity of $\mathrm{K}^{+}$channels. Notable examples are the heteromeric channels Kv1.1/Kv1.2 or Kv1.1/Kv1.4 that are localized in several structures within the nervous system (Wang et al., 1993, 1994; Sheng et al., 1994; D'Adamo et al., 1999). The need for such a large number of $\mathrm{K}^{+}$ channels that dampen cell excitability remains unclear.

The Kv1 family members exhibit diverse expression patterns in the central and peripheral nervous system and are found tightly clustered within distinct neuronal compartments (for a review see Trimmer and Rhodes, 2004). Knowledge of such precise targeting has important implications for defining the roles played by individual $\mathrm{Kv}$ channels in regulating neuronal function and in EA1 pathophysiology. In particular, Kv1.1 and Kv1.2 are expressed at the cerebellar pinceau, a structure composed of a number of basket cell terminals that embrace the Purkinje axon hillock and proximal axon segment (McNamara et al., 1993; Wang et al., 1993, 1994; Laube et al., 1996). A basket cell makes synaptic contact with several Purkinje cells. Patch-clamp recordings from cerebellar Purkinje cells have revealed that $\alpha$-DTX, a selective blocker of Kv1.1 and Kv1.2 K+ channels from basket cell presynaptic terminals, increases both the amplitude and frequency of spontaneous inhibitory postsynaptic currents (sIPSCs) mediated by $\gamma$-aminobutyric acid (GABA) A receptor activation (Southan and Robertson, 1998). These findings suggest that heteromeric channels Kv1.1/ Kv1.2, which are expressed at the presynaptic level of basket cells, modulate their excitability and the release of the neurotransmitter GABA onto Purkinje cells.

\subsubsection{EA1: Clinical Findings}

EA1 (OMIM: 160120) was clearly described during the mid-1970s by Van Dyke and colleagues (1975). It is characterized by constant myokymia and dramatic episodes of spastic contractions of the skeletal muscles of the head, arms, and legs with loss of both motor coordination and balance (for a review see D'Adamo et al., 2012; Pessia et al., 2012). During attacks, some individuals may experience vertigo, blurred vision, diplopia, 


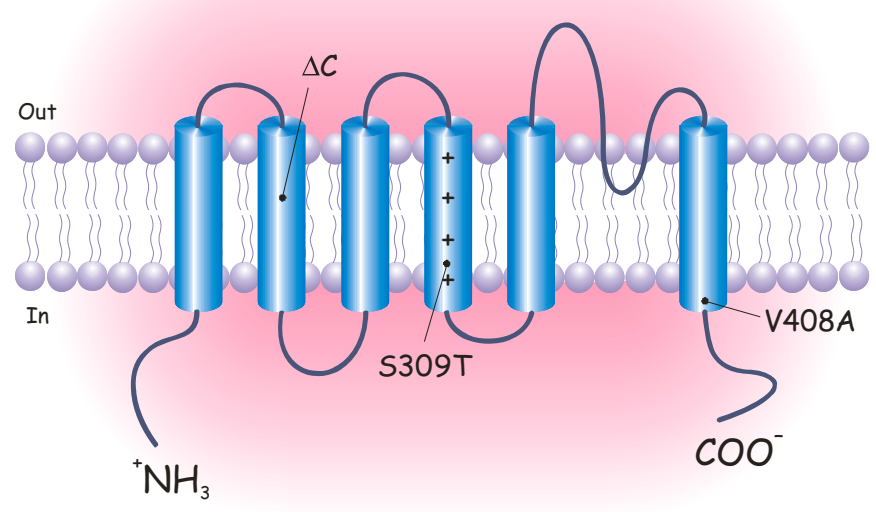

FIGURE 51.3 Schematic diagram illustrating the predicted secondary structure of a Kv1.1 subunit and the positions of the Kcna1 mutations harbored by animal models of episodic ataxia type 1 (EA1). A Kv1.1 subunit is composed of a voltage-sensing region (transmembrane [TM] 1 through TM4) and a pore region (TM5-H5-TM6), with the N- and C-termini residing inside the cell. A functional channel is formed by the assembly of four subunits. $\Delta \mathrm{C}$ refers to a deletion mutation identified in mceph/mceph mice: $m K v 1.1^{\Delta \mathrm{C} / \Delta \mathrm{C}}$ (Petersson et al., 2003); S309T is a missense mutation identified in autosomal-dominant myokymia and seizures rats: $r K v 1.1^{\text {S309T/+ }}$ (Ishida et al., 2012); V408A is a mutation identified in individuals with EA1 that was inserted into the murine Kcna1 to generate a knockin animal model of the disease: $m K v 1.1^{\text {V408A/+ }}$ (Herson et al., 2003).

nausea, headache, diaphoresis, clumsiness, stiffening of the body, and dysarthric speech. The duration of the attacks is brief, lasting seconds to minutes, although prolonged attacks of 5-12 h have been described (Lee et al., 2004). Some individuals experience severe ataxia more than 15 times per day, whereas others experience attacks less than once a month (Van Dyke et al., 1975). The first symptoms typically manifest during childhood (the first or second decade of life). A specific traumatic physical or emotional event may determine the onset and worsening of the disease (Imbrici et al., 2008). Attacks may be brought on by stimuli including fever, startle response, abrupt movements, vestibular caloric stimulation, emotional stress, anxiety, repeat knee bends, exercise, ingestion of caffeine, and riding a merry-go-round. Attacks may occur, for example, when the individual has had to suddenly alter course to avoid falling or a potential collision. High temperatures that occur after a hot bath or during use of a hairdryer may also precipitate attacks (Eunson et al., 2000).

Continuous myokymia manifests clinically during and between attacks as fine twitching of groups of muscles and intermittent cramps and stiffness. Usually, it is evident as a fine rippling in perioral or periorbital muscles and by lateral finger movements when the hands are held in a relaxed, prone position. The exposure of the forearm to warm or cold temperatures may increase or decrease, respectively, the spontaneous activity recorded from a hand muscle. In some individuals myokymic activity on an electromyograph (EMG) becomes apparent after the application of regional ischemia.

Since the first description of EA1 by Van Dyke and coworkers and the identification and characterization of mutations in the KCNA1 gene, the phenotypic spectrum of EA1 has widened considerably. Some individuals display attacks of difficult breathing, which can occur during ataxic episodes or as isolated episodes of an inability to inhale without wheezing (Shook et al., 2008). Others may also display cognitive dysfunctions that include severe receptive and expressive language delay, inability to learn to ride a bicycle, and the need to join life skill programs or attend schools for children with mild to moderate learning difficulties (Zuberi et al., 1999; Demos et al., 2009). Abnormal electroencephalograms (EEGs) have been observed in individuals with EA1 (Van Dyke et al., 1975; Zuberi et al., 1999; Lee et al., 2004). EEGs may be characterized by intermittent and generalized slow activity frequently intermingled with spikes. Zuberi and colleagues (1999) described a 3-year-old boy who presented with an ictal EEG with rhythmic, slow wave activity over the right hemisphere, which became spike-and-wave complexes that subsequently spread to the left hemisphere.

\subsubsection{EA1: Genetics and Molecular Pathogenesis}

EA1 is a disorder inherited in an autosomal-dominant manner. Linkage studies using several EA1 families have led to the discovery of a number of heterozygous point mutations in KCNA1 (Kv1.1) on chromosome 12p13 (Litt et al., 1994; Browne et al., 1994, 1995; Comu et al., 1996; Imbrici et al., 2008). To date KCNA1 is the only gene known to be associated with EA1, and more than 20 KCNA1 mutations have been identified by sequence analysis, which are distributed throughout the gene (for a review see D'Adamo et al., 2012). Symptom heterogeneity among individuals harboring the same mutation may reflect the interplay of nongenetic factors (Graves et al., 2010). Indeed, even identical twins harboring the same EA1 mutation displayed unexpectedly large differences in the severity and frequency of attacks (Graves et al., 2010).

The molecular mechanisms underlying EA1 have been established by determining the functional properties of wild-type (WT) and several mutant channels in Xenopus oocytes or mammalian cell lines (Adelman et al., 1995; D'Adamo et al., 1998; Zerr et al., 1998; D'Adamo et al., 1999; Zuberi et al., 1999; Eunson et al., 2000; Manganas et al., 2001; Cusimano et al., 2004; Imbrici et al., 2003, 2007, 2008, 2006, 2007, 2008, 2009). In particular, V408A channels display faster kinetics of current decay. Individuals with EA1 are heterozygous for a KCNA1 mutation, possessing a normal and a mutant allele that may 
be equally or differently expressed. Therefore, channels composed of WT and mutated subunits might be formed. The heterozygous channels composed of two Kv1.1 WT and two mutated subunits, linked as dimers, showed gating properties intermediate between channels formed from four normal or four mutated subunits at both the whole-cell and single channel level (D'Adamo et al., 1998). These results indicated that the degree of impairment of the delayed rectifier function of affected neurons is related to the type and number of mutated subunits that make up the tetrameric Kv1.1 channels (D'Adamo et al., 1998). Coexpression experiments also showed that some mutant subunits exert dominant negative effects on WT subunits, resulting in less than half the normal current, whereas others have virtually no effect (Zerr et al., 1998; D’Adamo et al., 1999; Rea et al., 2002; Imbrici et al., 2006, 2011; Graves et al., 2010).

Kv1.1, Kv1.2, and Kv1.4 are the most abundant subunits expressed in the central nervous system and are assembled to form heteromeric channels composed of Kv1.1/Kv1.2 and Kv1.1/Kv1.4 subunits. D'Adamo and colleagues (1999) demonstrated that EA1 mutations also alter the properties of these channels. This indicated that allelic variations in a single gene (KCNA1) also alter the function of other proteins that interact with Kv1.1 (D'Adamo et al., 1999; Imbrici et al., 2006, 2011). Overall, these studies demonstrated that allelic variations underlying EA1 impair channel function and reduce the outward $\mathrm{K}^{+}$flux through the channel but have highly variable effects on aspects of channel expression and gating.

Based on the body of molecular and neurophysiological evidence, D'Adamo and colleagues (1999) proposed that the reduced delayed rectifier function of EA1 heteromeric channels, comprised of Kv1.1 and Kv1.2 subunits, prolong the duration of action potentials at the cerebellar basket cell presynaptic terminals and enhance $\mathrm{Ca}^{2+}$ ion influx. Larger amounts of GABA were predicted to be released from basket cells terminals, reducing the ability of the relevant Purkinje cells to generate action potentials. As a result, the output of the entire cerebellum to the rest of the brain was expected to be markedly altered, leading to the cerebellar symptoms characteristic of EA1 syndrome (see Figure 7 in D'Adamo et al., 1999; D'Adamo et al., 2012).

\section{2 $\mathrm{MKV} 1.1^{\mathrm{V} 408 \mathrm{~A} /+}$ : A KNOCKIN MURINE MODEL OF EA1}

\subsubsection{Motor Deficits}

A murine model of EA1 ( $\left.m K v 1.1^{V 408 A /+}\right)$ has been generated by inserting a very conservative valine-to-alanine substitution (p.Val408Ala) in the mouse Kcna1(mKv1.1) by homologous recombination (Herson et al., 2003) (Figure 51.3). Heterozygous $m K v 1.1^{V 408 A /+}$ mice were

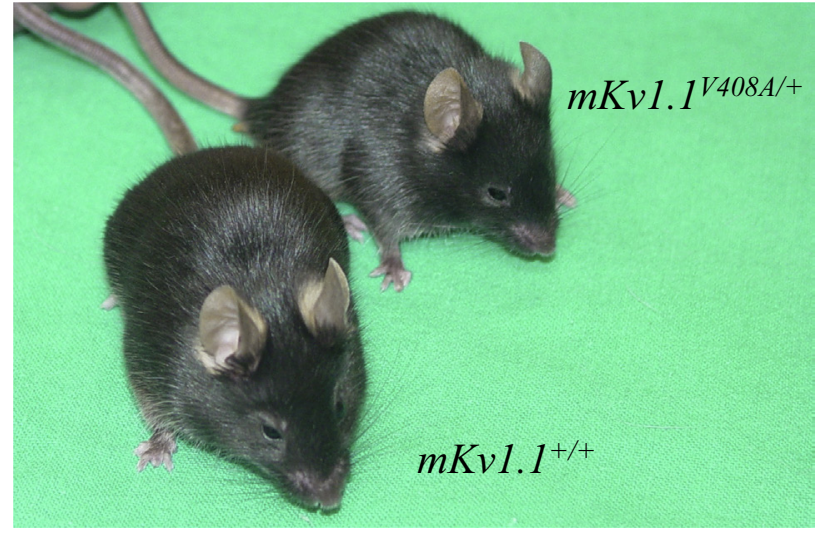

FIGURE 51.4 C57BL/6J mice expressing wild-type channels $\left(m \mathrm{Kv} 1.1^{+/+}\right)$or the heterozygous mutation V408A $\left(m \mathrm{Kv} 1.1^{\mathrm{V} 408 \mathrm{~A} /+}\right)$.

viable (Figure 51.4), whereas the homozygous V408A mutation was lethal - it caused death of $m K v 1.1^{V 408 A / V 408 A}$ mice between embryonic days 3 and 9. Interestingly, no homozygous KCNA1 mutations have been reported in humans, a condition that may be lethal as well. A rigorous evaluation of the motor coordination of $m K v 1.1^{V 408 \mathrm{~A} /+}$ mice performed using a balance beam, accelerating rotarod, or treadmill did not reveal any obvious abnormalities. Attacks of ataxic gait often are precipitated by stress in individuals with EA1. Indeed, a combination of exercise and administration of the $\beta$-adrenergic agonist isoproterenol $(10 \mathrm{mg} / \mathrm{kg})$ to $m K v 1.1^{\mathrm{V408A} /+}$ mice, a procedure that mimics stress-fear responses, reduced the latency of falls on a treadmill and increased the number of hind foot missteps while walking across a balance beam compared to WT animals (Herson et al., 2003). Acetazolamide pretreatment of $m K v 1.1^{V 408 \mathrm{~A} /+}$ mice significantly ameliorated their stress-induced motor coordination deficits. Acetazolamide is a carbonic-anhydrase (CA) inhibitor that is commonly used by medical practitioners to reduce the frequency and severity of attacks in responsive individuals with EA1. These results indicate that $m K v 1.1^{V 408 A /+}$ recapitulates some motor dysfunction and displays sensitivity to a typical pharmacological treatment.

\subsubsection{Cerebellar Deficits}

Whole-cell patch-clamp recordings of cerebellar Purkinje cells from brain slices showed strong sIPSCs mediated at $\mathrm{GABA}_{\mathrm{A}}$ receptors (Figure 51.5). sIPSCs resulted from GABA release from basket cell presynaptic terminals onto Purkinje cells. Both the frequency and amplitude of sIPSCs recorded from the Purkinje cells of $m K v 1.1^{V 408 A /+}$ mice were larger than those from the WT mice (Herson et al., 2003) (Figure 51.5). However, the shape (rise and decay time constants) of individual sIPSCs and the frequency and amplitude of miniature IPSCs did not differ between both genotypes. Paired recordings from a basket cell and a Purkinje cell, coupled synaptically, revealed 
(A)

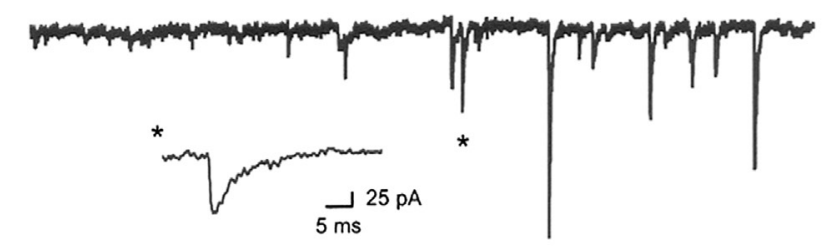

(B)

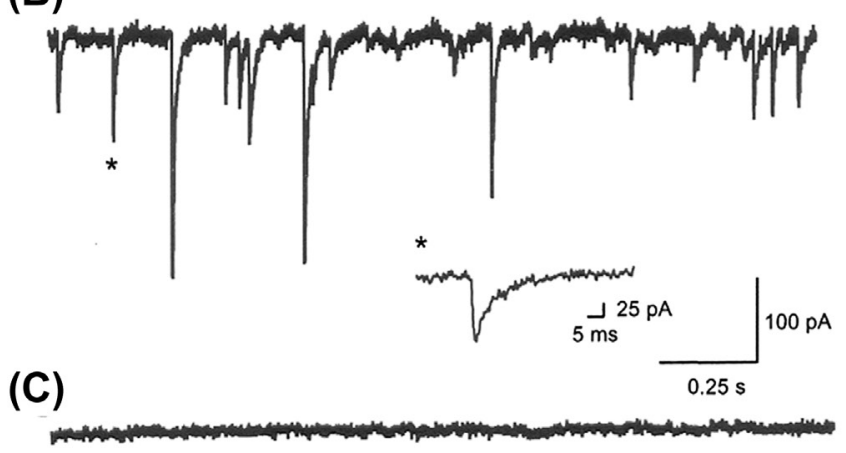

that the spontaneous firing frequency of $m K v 1.1^{V 408 \mathrm{~A} /+}$ basket cells was similar to that of WT cells (Herson et al., 2003). It is important to note that preliminary data showed that the action potential duration at half amplitude was broader in basket cell terminals of $m K v 1.1^{V 408 A /+}$ mice $(1.30 \pm 0.12 \mathrm{~ms} ; \mathrm{n}=10)$ than in WT mice $(0.90 \pm 0.04 \mathrm{~ms}$; $\mathrm{n}=4$ ) (Begun and Kullmann, 2011). This evidence clearly demonstrated that the p.Val408Ala mutation causes dysfunctions in a distinct circuitry that may underlie the cerebellar symptoms of the disease, in accordance with previously postulated pathogenic mechanisms underlying EA1 (see Figure 7 in D'Adamo et al., 1999).

\subsubsection{Neuromuscular Transmission Deficits}

The myelinated axon of the peripheral nervous system is subdivided into several specialized domains. They include, in order, the node of Ranvier, paranode, juxtaparanode, and internode. The juxtaparanodal region of myelinated axons expresses a macromolecular membrane complex composed of Kv1.1, Kv1.2, and their accessory subunit Kv $\beta 1.2$ (Vacher et al., 2008; Wang et al., 1993; Poliak et al., 1999). This macromolecular complex also has been found at the level of the axons branching in the central nervous system and peripheral nervous system (Tsaur et al.,1992; Wang et al., 1994). However, Kv1.1 channels are absent at both the end-plate and muscle fibers (Arroyo et al., 1999; Vacher et al., 2008; Zhou et al., 1998). Action potentials (APs) propagate rapidly in myelinated axons by saltatory conduction, although the role of Kv1.1 channels in this process is unclear. We recently investigated the neuromuscular transmission in $m K v 1.1^{V 408 \mathrm{~A} /+}$ mice and the susceptibility to physiologically relevant stressors
(D)

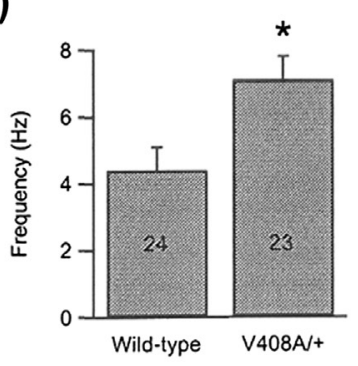

(E)

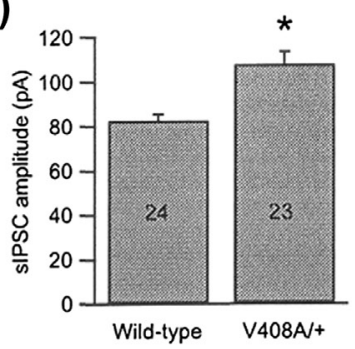

FIGURE 51.5 The Purkinje cells of $m K v 1.1^{\text {V408A/+ }}$ mice exhibit spontaneous inhibitory postsynaptic currents (sIPSCs) with increased frequency and amplitude. Representative 2-s segment of sIPSCs recorded from $m K v 1.1^{+/+}$ (wild-type) mice (A) and $m K v 1.1^{\text {V408A/+ }}$ (V408A/+) littermates (B) Inset: a single postsynaptic current measured at the time point indicated $\left({ }^{*}\right)$. (C) Application of $100-\mu \mathrm{M}$ picrotoxin to the cell in (B) completely blocked the postsynaptic currents. (D) Frequency of sIPSCs recorded $\left({ }^{*} \mathrm{P}<0.01\right.$; the number of experiments is indicated for each bar). (E) Mean amplitude of sIPSCs $\left({ }^{*} P<0.01\right)$. Reproduced from Herson et al. (2003) with permission.
(Brunetti et al., 2012). By using in vivo preparations of lateral gastrocnemius (LG) nerve-muscle from $m \mathrm{Kv} 1.1^{+/+}$ and $m K v 1.1^{V 408 A /+}$ mice, we observed that the mutant animals displayed spontaneous myokymic-like discharges consisting of repeated singlets, duplets, or multiplets (Figure 51.6). Thus, $m K v 1.1^{V 408 A /+}$ also recapitulates a hallmark of EA1, namely continuous myokymia (muscle twitching with a rippling appearance, intermittent cramps, and stiffness) (D'Adamo et al., 2012). To investigate the role played by the sciatic nerve in the myokymic activity observed in $m K v 1.1^{V 408 A /+}$ mice, $\mathrm{Ca}^{2+}$ signals were recorded ex vivo by fluorescent imaging of the sciatic nerve, which was previously loaded with the calcium sensitive dye fluo-3 acetoxymethyl (Lev-Ram and Ellismann, 1995). Twophoton laser scanning microscopy from the motor nerve revealed spontaneous $\mathrm{Ca}^{2+}$ signals that occurred abnormally only in preparations dissected from $m K v 1.1^{V 408 \mathrm{~A} /+}$ mice (Brunetti et al., 2012) (Figure 51.7). These results indicated that heterozygous V408A mutation in Kcna1 results in abnormal $\mathrm{Ca}^{2+}$ signals in sciatic nerves. Trains of high-frequency stimulations were applied to the LG motor nerve to mimic muscle fatigue. This procedure increased spontaneous EMG bursting activity by eliciting singlets, duplets, and multiplets from $m K v 1.1^{V 408 \mathrm{~A} /+}$. By contrast, the EMG activity in $m K v 1.1^{+/+}$remained silent before and after the tetanic stimulation and throughout the entire recovery period from fatigue (Brunetti et al., 2012). The EMG responses to ischemia and reperfusion, induced by temporarily occluding the artery and venous vessels supplying the LG nerve and muscle, were evaluated in $m K v 1.1^{+/+}$and $m K v 1.1^{V 408 A /+}$. In none of these conditions were after-discharges ever detected in $m \mathrm{Kv} 1.1^{+/+}$. However, ischemia elicited delayed activity consisting 


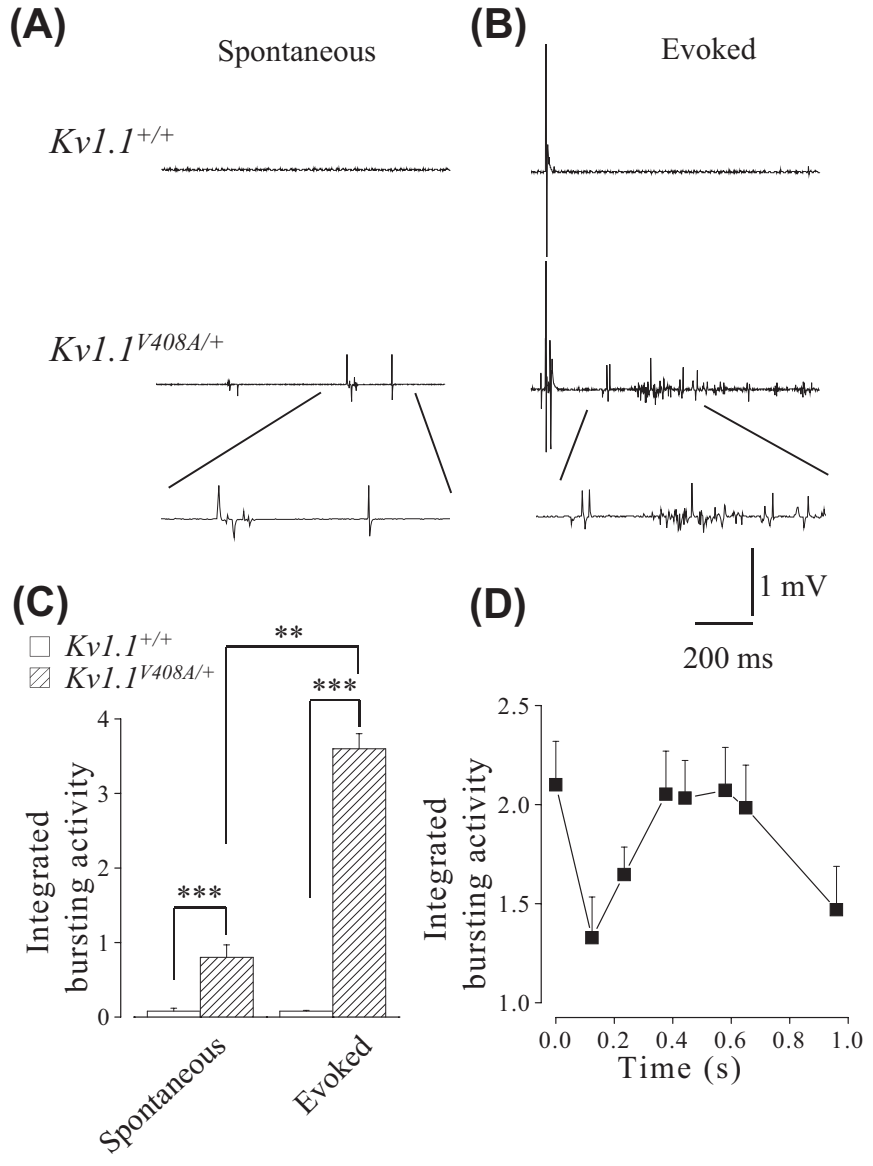

FIGURE $51.6 \quad m K v 1.1^{\mathrm{Vu08A} /+}$ mice display spontaneous muscle discharges. Electromyographic (EMG) recordings from lateral gastrocnemius (LG) muscles of $m K v 1.1^{+/+}$(top traces) and $m K v 1.1^{V 408 \mathrm{~A} /+}$ (bottom traces) showing spontaneous activity (A) and activity following the nerve-evoked compound muscle action potential (CMAP) (B). Enlargements of the traces are presented below to show the shape of the spontaneous and evoked repetitive muscle activity for $m K v 1.1^{\mathrm{Vu} 08 \mathrm{~A} /+}$ mice that was absent in all $m K v 1.1^{+/+}$mice tested. (C) Bar graph showing the averaged values of the integrated bursting activity normalized to the integrated CMAP (EMG/CMAP) either in resting conditions (spontaneous) or during the poststimulus periods (evoked) for both $m K v 1.1^{+/+}$ mice (open bars; $\mathrm{n}=10$ ) and $m K v 1.1^{V 408 A /+}$ mice (dashed bars; $\mathrm{n}=10$ ). Note that for $m K v 1.1^{V 408 A /+}$ mice, the evoked EMG activity is remarkably higher than its own spontaneous level of activity $\left({ }^{* *} P<0.01\right.$, ${ }_{* * *} P<0.001$ ). (D) Plot of the EMG activity evoked in $m K v 1.1^{V 408 \mathrm{~A} /+}$ mice as a function of time. The integrated values were calculated just after the CMAP and multiplied by 10 . Note that the single shock stimulation of the motor nerve elicited an immediate induction of EMG activity, followed by a second peak $400-600 \mathrm{msec}$ later that gradually decayed. Data are means \pm standard errors of the mean of 10 animals. Reproduced from Brunetti et al. (2012) with permission.

of singlets, duplets, and multiplets in $m K v 1.1^{\mathrm{V408A/+}}$. This bursting activity also was exacerbated by lower temperatures. Indeed, cooling induced poststimulus discharges from only $m K v 1.1^{V 408 A /+}$; these were characterized by the presence of randomly distributed singlets, duplets, and multiplets resembling those observed under fatigue and ischemia (Brunetti et al., 2012).
The distinct expression of Kv1.1 in axonal structures and the fact that spontaneous discharges are present despite severing the motor nerve supplying the $m K v 1.1^{V 408 \mathrm{~A} /+}$ muscle indicates that the sciatic nerve is certainly a site of origin of anomalous reexcitation. This represents a clear indication that myokymic activity in patients with EA1 also originates from hyperexcitability of the peripheral nerve endings. It also is consistent with previous neurophysiological investigations of patients with EA1. Indeed, individuals manifesting EA1 symptoms, including myokymia/neuromyotonia, and harboring different KCNA1 mutations displayed 100\% higher axonal superexcitability compared to healthy controls (Tomlinson et al., 2010).

In conclusion, the study by Brunetti and coworkers (2012) provided direct evidence indicating that the intrinsic hyperexcitability of the motor axon in $m \mathrm{Kv} 1.1^{\mathrm{V} 408 \mathrm{~A} /+}$ contributes to the myokymia/neuromyotonia phenotype, which is exacerbated by stressful events such as fatigue, ischemia, and lower temperature. The other key findings pointed out that: (1) Kv1.1 ${ }^{V 408 \mathrm{~A} /+}$ mice recapitulate the spontaneous and the stress-induced neuromuscular hyperexcitability observed in individuals with EA1; (2) dysfunction of Kv1.1 channels alters $\mathrm{Ca}^{2+}$ homeostasis in motor axons that are likely to contribute to spontaneous myokymic activity; (3) muscle fatigue contributes relatively more than peripheral nervous system fatigue to exacerbate myokymia/neuromyotonia; and (4) juxtaparanodal $\mathrm{K}^{+}$channels composed of Kv1.1 subunits tone down nerve excitability during both fatigue and ischemic insult.

\subsubsection{Optical and Electron Microscopy Analysis of LG Muscle and Nerve Preparations}

The repetitive firing of the $m K v 1.1^{V 408 A /+}$ muscle fibers combined with clinical findings reporting enlargement of gastrocnemius fiber type I and II diameters in some individuals with EA1 (Van Dyke et al., 1975) prompted us to perform morphological studies of LG muscle sections and nerves derived from $m K v 1.1^{+/+}$and $m K v 1.1^{V 408 A /+}$ adult animals. LG muscles dissected from these animals had similar muscle mass. Histograms of the frequency distribution for fiber type I and type II diameters were similar for LG muscles from both $m \mathrm{Kv} 1.1^{+/+}$and $m K v 1.1^{V 408 \mathrm{~A} /+}$ animals. Neither the capillary density nor the capillary diameter of LG muscles differed between both genotypes. Finally, the electron microcopy analysis of the neuromuscular junction also revealed no obvious differences (Brunetti et al., 2012). Taken together, these findings imply that the spontaneous and fatigue-induced LG neuromuscular hyperexcitability are not sufficient to result in major morphological abnormalities in $m K v 1.1^{\mathrm{V408A/+}} \mathrm{LG}$ muscles. 
(A)

$$
K v 1.1^{+/+}
$$

(B)

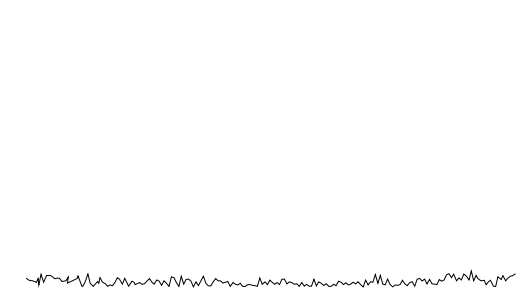

(C)

$$
K v 1.1^{+/+}
$$

(D)

『 Kv1.1 $1408 A /+$
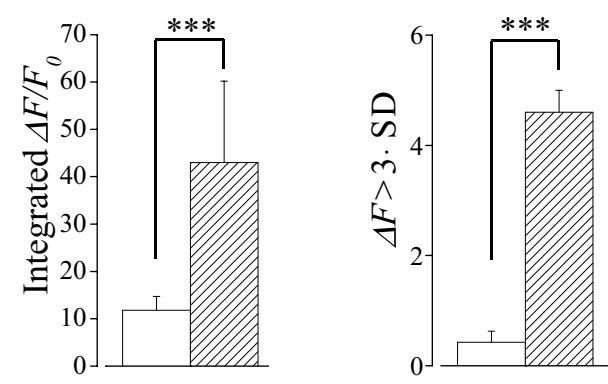

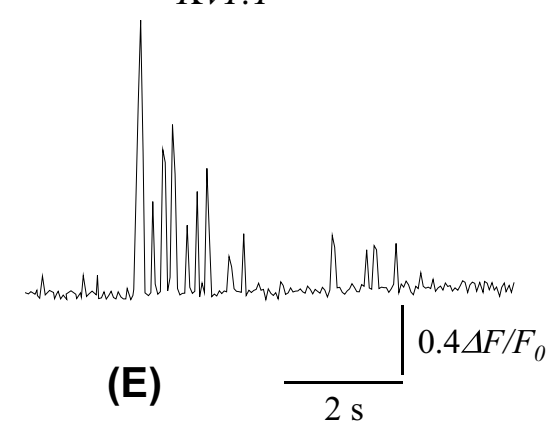

FIGURE $51.7 \quad \mathrm{Ca}^{2+}$ signals occur spontaneously within the sciatic nerve of $m K v 1.1^{\mathrm{V} 408 \mathrm{~A} /+}$ mice. Representative traces showing changes in $\mathrm{Ca}^{2+}$ signals over time recorded from the sciatic nerve of $m K v 1.1^{+/+}(\mathrm{A})$ and $m K v 1.1^{\mathrm{V} 408 \mathrm{~A} /+}$ mice (B) (C) Bar graph showing the averaged values of the integrated fluorescence signals $\left(\Delta F / F_{o}\right)$ for both $m K v 1.1^{+/+}$(open bars; $\mathrm{n}=5$ ) and $m K v 1.1^{V 408 A /+}$ mice (dashed bars; $n=5)$. Note that the integrated $\mathrm{Ca}^{2+}$ fluorescence recorded in resting conditions from the sciatic nerves of $m K v 1.1^{V 408 A /+}$ mice is remarkably higher than in normal animals (***P<0.001, analysis of variance). (D) Bar graph showing the number of $\Delta F$ data points that lay outside three standard deviations from the mean fluorescence recorded from the sciatic nerves of both $m K v 1.1^{+/+}$(open bars) and $m K v 1.1^{V 408 A /+}$ mice (dashed bars) ${ }^{* * *} P<0.001, \chi^{2}$ analysis). (E) Representative series of two-photon imaging acquired before (top panel) and during the occurrence of abnormal $\mathrm{Ca}^{2+}$ signals (bottom panel boxed area) in a small branch of the sciatic nerve visualized within a lateral gastrocnemius muscle dissected from a $m K v 1.1^{V 408 \mathrm{~A} /+}$ mouse.

\subsection{MKV1.1-/-: A KNOCKOUT MURINE MODEL OF EA1}

\subsubsection{Motor Deficits}

$m K v 1.1^{-/-}$was generated by ablating Kcna1 through homologous recombination in embryonic stem cells (Smart et al., 1998). These null mice showed a defect in their ability to maintain balance on a thin stationary rod, unrelated to seizures (Zhang et al., 1999). Surprisingly, the mutants failed to show a defect in their ability to remain on the rotating rod. Neuromyotonia and an inability to maintain an axial position was observed when they were forced to swim in cold water. In particular, when lifted out of the water and placed on a dry platform the $m \mathrm{Kv} 1.1^{-/-}$mouse fell on its side and exhibited severe neuromyotonia. This stress-induced motor dysfunction also resulted in generalized body tremors. The tremors were reduced as time progressed, but as the animal started to walk, its movements were staggering and ataxic (Zhou et al., 1998).

\subsubsection{Cerebellar Deficits}

Whole-cell patch-clamp recordings of sIPSC s were made from Purkinje cells in thin cerebellar slices from $m K v 1.1^{-/-}$ mice and WT littermates. These recordings showed that Kv1.1 ablation increased remarkably the frequency of the sIPSCs, without accompanying changes in the mean and variance of the amplitude distribution. This represented a noteworthy difference with $m K v 1.1^{V 408 A /+}$, whereby both the frequency and amplitude of sIPSCs recorded from the Purkinje cells were larger than those in the WT (Herson et al., 2003). Spontaneous firing rate of the $m \mathrm{Kv}_{1} .1^{-/-}$basket cells was unaltered, and evoked IPSCs did not show multiple activity in the mutants. This study suggested that the Kv1.1-null mutation results in a persistent elevation of the tonic inhibitory tone on the cerebellar Purkinje cell by enhancing the excitability of the basket cells and by selectively enhancing the likelihood of action potential propagation past axonal branch points (Zang et al., 1999).

\subsubsection{Neuromuscular Transmission Deficits}

The neuromuscular transmission of $m K v 1.1^{-/-}$has been investigated using phrenic nerve-diaphragm muscle preparations ex vivo (Zhou et al., 1998, 1999; Zhou and Chiu, 2001). These animals displayed subtle discharges in diaphragm muscles (Zhou et al., 1998, 1999). Nevertheless, the phenotype could be clearly unmasked by low temperatures $\left(-20^{\circ} \mathrm{C}\right)$, which resulted in stimulus-induced repetitive discharges (Zhou et al., 1998, 1999). The effects of additional stressors such as fatigue and ischemia were not investigated. Zhou and colleagues (1999) concluded that the temperature-induced neuromuscular hyperexcitability in the mutant occurs solely as a result of losing $\mathrm{K}^{+}$channels normally concealed in the segment just preceding the terminal and not elsewhere in the myelinated fiber. This hypothesis was corroborated by computer simulations showing that lack of juxtaparanodal Kv1.1 channels leads to reentrant excitation of nodes due to nerve backfiring at axon transition 
zones in myelinated nerve terminals (Zhou et al., 1999). However, this conclusion is somehow inconsistent with (1) the slower repolarization phases of the compound action potentials measured from sciatic nerves of $m K_{v} 1.1^{-/-}$mice (Smart et al., 1998), (2) the abnormal $\mathrm{Ca}^{2+}$ signals detected elsewhere in sciatic nerves of $m K v 1.1^{\text {V408A/+ }}$ mice, and (3) EA1 clinical findings (Brunt and Van Weerden, 1990; Tomlinson et al., 2010). Overall, this evidence indicated that the nodes along the fiber also are affected by Kv1.1 channel dysfunction. Interestingly, computer simulations showed that cooling slows down $\mathrm{K}^{+}$clearing from peri-internodal space. This mechanism may cause more pronounced after-depolarizations in $m K v 1.1^{-/-}$(Zhou and Chiu, 2001) and contribute to temperature enhancements of the myokymic activity from $m K v 1.1^{V 408 A /+}$. Ultimately, such abnormal transmission of signals occurring in the phrenic nerve of $m \mathrm{Kv} 1.1^{-/-}$ mice may underlie the respiratory symptoms reported in some individuals with EA1 who displayed attacks of difficult breathing during episodes of ataxia or isolated inability to inhale without wheezing (Shook et al., 2008).

\subsubsection{Epilepsy}

Epilepsy is overrepresented in EA1 and neuronal circuit dysfunction within the hippocampus and has been postulated to play a role in seizures and cognitive dysfunction associated with EA1. The hippocampus is a major brain region belonging to the limbic system, which plays an important role in the consolidation of information and in spatial memory. This area is often the focus of epileptic seizures. In the hippocampus, Kv1.1, Kv1.2, and Kv1.4 are found in Schaffer collateral axons and are highly expressed in axons and terminals of the medial perforant path in the middle third of the molecular layer of the dentate gyrus. In CA3, Kv1.1, Kv1.4, and Kv $\beta 1.1$ subunits are expressed in mossy fiber boutons (swellings of mossy fiber axons) that form en passant synapses with pyramidal neurons. This macromolecular channel complex regulates the activity-dependent spike broadening of hippocampal mossy fiber boutons and, consequently, the amount of neurotransmitter released during highfrequency stimuli (Geiger and Jonas, 2000).

$m K v 1.1^{-/-}$mice display morphology characteristic of the epileptic hippocampus (Rho et al., 1999), with neural loss, astrocytosis, and mossy fiber sprouting. Mice lacking these channels also exhibit frequent spontaneous seizures throughout adult life, although the intrinsic passive properties of CA3 pyramidal cells are normal. Antidromic action potentials were recruited at lower thresholds in $m \mathrm{Kv} 1.1^{-/-}$slices, and mossy fiber stimulation triggered synaptically mediated long-latency epileptiform burst discharges. This data indicates that loss of Kv1.1 results in increased excitability in the CA3 recurrent axon collateral system, perhaps contributing to the limbic and tonic-clonic components of the observed epileptic phenotype of EA1 (Smart et al., 1998). In vitro extracellular recordings were recently performed using a multielectrode array to characterize spontaneous sharp waves and high-frequency oscillations in $m \mathrm{Kv} 1.1^{-/-}$hippocampi. This study showed that the mossy fibers and medial perforant path axons of $m \mathrm{Kv} 1.1^{-/-}$were hyperexcitable and produced greater pre- and postsynaptic responses with reduced paired-pulse ratios. Microdissection of $m \mathrm{Kv} 1.1^{-/-}$mossy fibers and perforant paths ameliorated the abnormal oscillatory pattern and improved spike timing. Blockade of Kv1.1 channels with dendrotoxin-K reproduced these effects in WT slices. These findings suggested that loss of Kv1.1 enhances synaptic release in the CA3 region, which reduces the precision of spike timing in individual neurons, leading to disorganization of network oscillatory activity and promoting the emergence of fast ripples (Simeone et al., 2013).

Comparatively, the $m \mathrm{Kv} 1.1^{-/-}$phenotype is apparently less severe than the $m K v 1.1^{V 408 A / V 408 A}$ phenotype. Indeed, the latter homozygous mutation is embryonically lethal (Herson et al., 2003), whereas the former results in viable animals at birth, although $50 \%$ of them die suddenly 3-5weeks later (Smart et al., 1998). It is conceivable that Kcna1 ablation might lead to a number of unpredictable compensatory effects, including the formation of Kv1.2 homomultimers or the upregulation of other $\mathrm{K}^{+}$channels (Wang et al., 1993). The dysfunction of the macromolecular membrane complex, however, composed of Kv1.1 ${ }^{\mathrm{V}} 08 \mathrm{~A}$ and Kv1.2 (D'Adamo et al., 1999) or Kv1.4 subunits (Imbrici et al., 2011), and the lack of compensatory effects may account, at least in part, for the differences in phenotype severity between $m \mathrm{Kv} 1.1^{\mathrm{V} 408 \mathrm{~A} /+}$ and $\mathrm{Kv} 1.1^{-/-}$mice. This might explain also why remarkable cooling is always required to unmask the neuromuscular phenotype in mKv1.1-/- mice (Zhou et al., 1998, 1999; Zhou and Chiu, 2001), whereas $m K v 1.1^{V 408 \mathrm{~A} /+}$ mice also display myokymia at $37^{\circ} \mathrm{C}$. Although the $m \mathrm{Kv} 1 \cdot 1^{-/-}$animal model does not faithfully recapitulate the EA1 phenotype, it does display some remarkable similarities with $m \mathrm{Kv} 1.1^{\mathrm{V} 408 \mathrm{~A} /+}$.

\subsection{RKv1.1 ${ }^{\text {S309T/+}: ~ A ~ R A T ~ M O D E L ~ O F ~ E A 1 ~}$}

N-ethyl-N-nitrosourea mutagenesis has been widely used to generate animal models of human diseases. An $\mathrm{N}$-ethyl-N-nitrosourea-mutagenized rat strain has been recently generated and named autosomal-dominant myokymia and seizures rats (Ishida et al., 2012). Genetic analysis of these animals resulted in the identification of the missense mutation S309T in rat Kcna1 (rKv1.1 $\left.{ }^{S 309 T /+}\right)$. The affected amino acid residue resides in the voltage-sensor domain TM4 of $r K v 1.1$ channels (Figure 51.3). The coinjection of WT and S309T complementary RNA (1:1 ratio) resulted in reduced outward currents, with an approximately 
$80 \%$ smaller amplitude than that of WT-injected oocytes, suggesting a dominant-negative effect of the S309T subunit on the function of heterotetrameric Kv1.1 channels. Western blot analysis showed equivalent Kv1.1 protein expression levels among the three groups (WT, S309T, and coinjected WT:S309T [1:1]) of oocytes (Ishida et al., 2012).

Heterozygous $r K v 1.1^{5309 T /+}$ rats appeared swollen and showed slightly increased body weight from 10 to 13 weeks of age that coincided with severe twitching behavior. Muscle twitching was characterized by coordinated muscle contraction of the eyelid, the neck, and the extremities and could be observed from 6 weeks of age. Startle responses to sudden acoustic stimuli also were evident. From 16 weeks of age their body weight significantly decreased, coinciding with severe periodic seizures, and by 30 weeks of age, $84 \%$ of $r$ Kv1.1 $1309 T /+$ rats had died. Postmortem examination showed no obvious morphological abnormalities in any examined tissues from $r K v 1.1^{5309 T /+}$ rats (Ishida et al., 2012). EMG recordings of forelimb and hind limb muscles showed spontaneous rhythmic myokymic discharges $(7 \mathrm{~Hz})$. Cooling induced severe neuromyotonia, ataxia, and aberrant spike-and-wave discharges $(2-3 \mathrm{~Hz})$ associated with clonus behaviors. $r K v 1.1^{S 309 T /+}$ rats also exhibited spontaneous convulsive seizures from 10 to 16 weeks of age, aggravated by stress (changing cages or handling the animal). Seizures were characterized by initial sudden falling down or jerking of the entire body or the extremities as clonic phase, occasionally with a tonic phase with stiffening of the entire body. Cortical and hippocampal EEG recordings identified aberrant large spike activity associated with falling-down behavior, low-voltage fast wave discharges detected during the tonic stage, and spikeand-wave discharges $(2 \mathrm{~Hz})$ detected during the clonic convulsive stage (Ishida et al., 2012). The behavioral phenotypes and abnormal discharge patterns in $r$ Kv1.1 $1^{\text {S309T/+ }}$ rats are similar to other rodent models of temporal lobe epilepsy. Carbamazepine administration ameliorated seizures. Homozygous $r K v 1.1^{\text {S309T/S309T }}$ rats were viable but displayed dramatic developmental impairments, tremors, motor incoordination, and spontaneous convulsive seizures. This phenotype progressively worsens with age, and all homozygous rats died prematurely, with a mean lifetime of 16 days (Ishida et al., 2012). The videos related to this animal model of EA1 are available online at http:/ /dx.doi.org/10.1016/j.brainres.2011.11.023.

\section{$51.5 \mathrm{MKv1.1} \triangle \mathrm{C} / \Delta \mathrm{C}:$ A MEGENCEPHALY MOUSE MUTANT DISPLAYING ATAXIA AND SEIZURES}

The megencephaly mice, mceph/mceph, are characterized by increased brain volume, hypertrophic brain cells, and slight hippocampal astrocytosis. They display a complex behavioral phenotype. Of relevance for EA1 are motor deficits such as shakiness in gait, periods of static immobilization, a strange posture sitting up, a remarkable sensitivity to sound, jittering, fluctuating hemiplegic symptoms, uncontrolled grooming, partial cramps, seizures when running, rearing, and falling. Complex partial seizures and postanesthetic tonic-clonic seizures are also overt (Donahue et al., 1996). The expression of molecules of the insulin-like growth factor system, transforming growth factor- $\beta 1$ (Petersson et al., 1999) as well as several trophic neuropeptides (Petersson et al., 2000) are significantly altered in the mceph/mceph mouse brain. Remarkably, an 11-base pair deletion in the Kcna1 gene of mceph/ mceph mice has been identified using a positional cloning approach (Petersson et al., 2003). The mutation leads to a frame shift and to a premature stop codon that was predicted to truncate the protein at amino acid 230 (of 495) (Figure 51.3). Therefore, mceph/mceph mice express Kv1.1 subunits lacking the last five TMs and the C-terminal domain: $m K v 1.1^{\Delta \mathrm{C} / \Delta \mathrm{C}}$. The absence of $\mathrm{Kv} 1.1$ channels with intact C-terminal domains was confirmed by performing Western blotting analysis of whole-protein extracts from brain. The expression of the remaining $\mathrm{N}$-terminal domain, first TM, and extracellular loop may be responsible for the decreased amounts of Kv1.2 and Kv1.3 proteins that were detected in the hippocampus of mceph/mceph mice. Electrophysiological investigations from mceph/ mceph brain slices revealed hippocampal hyperexcitability consistent with limbic status epilepticus (Petersson et al., 2003). Interestingly, a mutation that results in truncation of the C-terminal domain of Kv1.1 channels (R417stop) has also been found in an EA1 proband displaying episodes of ataxia precipitated by exercise, stress, startle, and high temperature such as occurs after a hot bath or when using a hairdryer (Eunson et al., 2000).

\subsection{CONCLUDING REMARKS}

Although important advances have been made in understanding the pathophysiology of EA1 using valuable animal models, some crucial information still remains elusive. In particular, the mechanisms underlying the central and peripheral neurological alterations resulting in EA1 symptoms are unclear. Remarkably, what determines the episodic nature of ataxia and epilepsy and why attacks are triggered by physiological stresses that stimulate otherwise normal responses in healthy subjects is unknown. Finding an answer to all these puzzling questions will be the challenge for future studies of EA1 and common paroxysomal disorders, including idiopathic epilepsy and migraine. Using higher-resolution technologies, biophysicists are shedding new light on the molecular machinery of channel gating, and physiologists are clarifying their normal 
location and functional roles. The knowledge resulting from these valuable studies is crucial to directing an exhaustive functional characterization of variants associated with CPs that, in turn, will allow for the full understanding of how mutations in ion channel genes cause disease. The detailed genetic, behavioral, and electrophysiological analysis of mice carrying naturally or artificially introduced mutations in ion channel genes is certainly providing important information about the causes of several human diseases and setting up the basis for new strategies of treatment. More broadly, however, investigations using these animal models are of crucial importance to understanding the physiological workings of the human body.

\section{Acknowledgments}

This work was supported by Telethon (GGP11188); Ministero della Salute (GR-2009-1580433); MIUR-PRIN 20108WT59Y_004; and Fondazione Cassa di Risparmio di Perugia.

\section{References}

Adelman, J.P., et al., 1995. Episodic ataxia results from voltage-dependent potassium channels with altered functions. Neuron 15, 1449-1454.

Arroyo, E.J., et al., 1999. Myelinating Schwann cells determine the internodal localization of Kv1.1, Kv1.2, Kvbeta2, and Caspr. J. Neurocytol. 28, 333-347.

Begun, R., Kullmann, D.M., 2011. Altered presynaptic spike properties in cerebellar basket cells in a mouse model of episodic ataxia type 1. Soc. Neurosci. Abst. 447, 15/E26.

Browne, D.L., et al., 1994. Episodic ataxia/myokymia syndrome is associated with point mutations in the human potassium channel gene, KCNA1. Nat. Genet. 8, 136-140.

Browne, D.L., et al., 1995. Identification of two new KCNA1 mutations in episodic ataxia/myokymia families. Hum. Mol. Genet. 4, 1671-1672.

Brunetti, O., et al., 2012. Kv1.1 knock-in ataxic mice exhibit spontaneous myokymic activity exacerbated by fatigue, ischemia and low temperature. Neurobiol. Dis. 47 (3), 310-321.

Brunt, E.R.P., van Weerden, T.W., 1990. Familial paroxysmal kinesigenic ataxia and continuous myokymia. Brain 113, 1361-1382.

Comu, S., et al., 1996. Episodic ataxia and myokymia syndrome: a new mutation of potassium channel gene Kv1.1. Ann. Neurol. 40 (4), 684-687.

Cusimano, A., et al., 2004. An episodic ataxia type-1 mutation in the S1 segment sensitises the hKv1.1 potassium channel to extracellular $\mathrm{Zn}^{2+}$. FEBS Lett. 576, 237-244.

D'Adamo, M.C., et al., 1998. Episodic ataxia type-1 mutations in the $\mathrm{hKv} 1.1$ cytoplasmic pore region alter the gating properties of the channel. EMBO J. 17, 1200-1207.

D'Adamo, M.C., et al., 1999. Mutations in the KCNA1 gene associated with episodic ataxia type-1 syndrome impair heteromeric voltagegated $\mathrm{K}^{+}$channel function. FASEB J. 13, 1335-1345.

D'Adamo, M.C., et al., 2012. Episodic ataxia type 1. In: Pagon, R.A., Bird, T.C., Dolan, C.R., Stephens, K. (Eds.), GeneReviews, second ed. University of Washington, Seattle (WA). Internet.

Demos, M.K., et al., 2009. A novel KCNA1 mutation associated with global delay and persistent cerebellar dysfunction. Mov. Disord. 24, 778-782.

Donahue, L.R., et al., 1996. Megencephaly: a new mouse mutation on chromosome 6 that causes hypertrophy of the brain. Mamm. Genome 7, 871-876.
Eunson, L.H., et al., 2000. Clinical, genetic, and expression studies of mutations in the potassium channel gene KCNA1 reveal new phenotypic variability. Ann. Neurol. 48, 647-656.

Geiger, J.R., Jonas, P., 2000. Dynamic control of presynaptic $\mathrm{Ca}^{2+}$ inflow by fast-inactivating $\mathrm{K}^{+}$channels in hippocampal mossy fiber boutons. Neuron 28, 927-939.

Graves, T.D., et al., 2010. Nongenetic factors influence severity of episodic ataxia type 1 in monozygotic twins. Neurology 75, 367-372.

Gutman, G.A., Chandy, K.G., 1993. Nomenclature of mammalian voltage-dependent potassium channel genes. Semin. Neurosci. 5, 101-106.

Herson, P.S., et al., 2003. A mouse model of episodic ataxia type-1. Nat. Neurosci. 6, 378-383.

Imbrici, P., et al., 2003. Functional characterization of an episodic ataxia type-1 mutation occurring in the S1 segment of hKv1.1 channels. Pflugers. Arch. EJP. 446, 373-379.

Imbrici, P., et al., 2006. Episodic ataxia type 1 mutations in the KCNA1 gene impair the fast inactivation properties of the human $\mathrm{K}^{+}$chan-

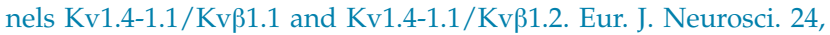
3073-3083.

Imbrici, P., et al., 2007. Episodic ataxia type 1 mutation F184C alters $\mathrm{Zn}^{2+}$-induced modulation of the human $\mathrm{K}^{+}$channel Kv1.4-Kv1.1/ Kvß1.1. Am. J. Physiol. Cell. Physiol. 292, C778-C787.

Imbrici, P., et al., 2008. A novel KCNA1 mutation identified in an Italian family affected by episodic ataxia type 1 . Neuroscience 157, $577-587$.

Imbrici, P., et al., 2009. Contributions of the central hydrophobic residue in the PXP motif of voltage-dependent $\mathrm{K}^{+}$channels to $\mathrm{S} 6$ flexibility and gating properties. Channels (Austin) 3, 39-45.

Imbrici, P., et al., 2011. Episodic ataxia type 1 mutations affect fast inactivation of $\mathrm{K}^{+}$channels by a reduction in either subunit surface expression or affinity for inactivation domain. Am. J. Physiol. Cell. Physiol. 300 (6), C1314-C1322.

Isacoff, E.Y., et al., 1990. Evidence for the formation of heteromultimeric potassium channels in Xenopus oocytes. Nature 345, 530-534.

Ishida, S., et al., 2012. Kcna1-mutant rats dominantly display myokymia, neuromyotonia and spontaneous epileptic seizures. Brain Res. 30 (1435), 154-166.

Jan, Y.N., et al., 1977. Two mutations of synaptic transmission in Drosophila. Proc. R. Soc. Lond. B Biol. Sci. 198, 87-108.

Jiang, Y., et al., 2003a. X-ray structure of a voltage-dependent $\mathrm{K}^{+}$channel. Nature $423,33-41$.

Jiang, Y., et al., 2003b. The principle of gating charge movement in a voltage-dependent $\mathrm{K}^{+}$channel. Nature 423, 42-48.

Laube, G., et al., 1996. Ultrastructural localization of Shaker-related potassium channel subunits and synapse-associated protein 90 to separate-like junctions in rat cerebellar pinceaux. Brain Res. Mol. Brain Res. 42, 51-61.

Lee, H.Y., et al., 2004. The gene for paroxysmal non-kinesigenic dyskinesia encodes an enzyme in a stress response pathway. Hum. Mol. Genet. 13, 3161-3170.

Lev-Ram, V., Ellisman, M.H., 1995. Axonal activation-induced calcium transients in myelinating schwann cells, sources, and mechanisms. J. Neurosci. 4, 2628-2637.

Litt, M., et al., 1994. A gene for episodic ataxia/myokymia maps to chromosome 12p13. Am. J. Hum. Genet. 55, 702-709.

Manganas, L.N., et al., 2001. Episodic ataxia type-1 mutations in the Kv1.1 potassium channel display distinct folding and intracellular trafficking properties. J. Biol. Chem. 276, 49427-49434.

McNamara, N.M., et al., 1993. Prominent location of a $\mathrm{K}^{+}$channel containing the alpha subunit $\mathrm{Kv} 1.2$ in the basket cell nerve terminals of rat cerebellum. Neuroscience 57 (4), 1039-1045.

Pessia, M., 2004. Ion channels and electrical activity. In: Davies, R. Wayne, Morris, Brian J. (Eds.), Molecular Biology of the Neuron, second ed. Oxford University Press, UK, pp. 103-137. 
Pessia, M., et al., 2012. The neurobiology of episodic ataxia type 1: a shaker-like $\mathrm{K}^{+}$channel disorder. In: Hong, Sung Hoi (Ed.), Ataxia: Causes, Symptoms and Treatment. NOVA SCIENCE, New York USA, pp. 47-75.

Petersson, S., et al., 1999. The megencephaly mouse has disturbances in the insulin-like growth factor (IGF) system. Mol. Brain Res. 72, 80-88.

Petersson, S., et al., 2000. Expression of cholecystokinin, enkephalin, galanin and neuropeptide $\mathrm{Y}$ is dramatically changed in the brain of the megencephaly mouse. Neuroscience 100, 297-317.

Petersson, S., et al., 2003. Truncation of the Shaker-like voltage-gated potassium channel, Kv1.1, causes megencephaly. Eur. J. Neurosci. 18 (12), 3231-3240.

Poliak, S., et al., 1999. Caspr 2 , a new member of the neurexin superfamily, is localized at the juxtaparanodes of myelinated axons and associates with $\mathrm{K}^{+}$channels. Neuron 24, 1037-1047.

Rea, R., et al., 2002. Variable $\mathrm{K}^{+}$channel subunit dysfunction in inherited mutations of KCNA1. J. Physiol. 538, 5-23.

Rho, J.M., et al., 1999. Developmental seizure susceptibility of Kv1.1 potassium channel knockout mice. Dev. Neurosci. 21, 320-327.

Ruppersberg, J.P., et al., 1990. Heteromultimeric channels formed by rat brain potassium-channel proteins. Nature 345, 535-537.

Sheng, M., et al., 1994. Contrasting subcellular localization of the Kv1.2 $\mathrm{K}^{+}$channel subunit in different neurons of rat brain. J. Neurosci. 14, 2408-2417.

Shook, S.J., et al., 2008. Novel mutation in KCNA1 causes episodic ataxia with paroxysmal dyspnea. Muscle Nerve 37, 399-402.

Simeone, T.A., et al., March 2, 2013. Loss of the Kv1.1 potassium channel promotes pathologic sharp waves and high frequency oscillations in in vitro hippocampal slices. Neurobiol. Dis. pii: S0969-9961(13) 00071-5.

Smart, S.L., et al., 1998. Deletion of the Kv1.1 potassium channel causes epilepsy in mice. Neuron 20 (4), 809-819.

Southan, A.P., Robertson, B., 1998. Patch-clamp recordings form cerebellar basket cell bodies and their presynaptic terminals reveal an asymmetric distribution of voltage-gated potassium channels. J. Neurosci. 18, 948-955.

Tanouye, M.A., Ferrus, A., 1985. Action potentials in normal and Shaker mutant drosophila. J. Neurogenet. 2, 253-271.
Tempel, B.L., et al., 1987. Sequence of a probable potassium channel component encoded at Shaker locus of Drosophila. Science 237, 770-775.

Tomlinson, S.E., et al., 2010. Nerve excitability studies characterize Kv1.1 fast potassium channel dysfunction in patients with episodic ataxia type 1. Brain 133 (Pt 12), 3530-3540.

Trimmer, J.S., Rhodes, K.J., 2004. Localization of voltage-gated ion channels in mammalian brain. Annu. Rev. Physiol. 66, 477-519.

Tsaur, M.L., et al., 1992. Differential expression of $\mathrm{K}^{+}$channel mRNAs in the rat brain and down-regulation in the hippocampus following seizures. Neuron 8, 1055-1067.

Vacher, H., et al., 2008. Localization and targeting of voltage-dependent ion channels in mammalian central neurons. Physiol. Rev. 88, 1407-1447.

Van Dyke, D.H., et al., 1975. Hereditary myokymia and periodic ataxia. J. Neurol. Sci. 25, 109-118.

Wang, H., et al., 1993. Heteromultimeric $\mathrm{K}^{+}$channels in terminal and juxtaparanodal regions of neurons. Nature 365, 75-79.

Wang, H., et al., 1994. Localization of Kv1.1 and Kv1.2, two $\mathrm{K}^{+}$channel proteins, to synaptic terminals, somata, and dendrites in the mouse brain. J. Neurosci. 14, 4588-4599.

Zerr, P., et al., 1998. Episodic ataxia mutations in Kv1.1 alter potassium channel function by dominant negative effects or haploinsufficiency. J. Neurosci. 18, 2842-2848.

Zhang, C.L., et al., 1999. Specific alteration of spontaneous GABAergic inhibition in cerebellar purkinje cells in mice lacking the potassium channel Kv1.1. J. Neurosci. 19 (8), 2852-2864.

Zhou, L., et al., 1998. Temperature-sensitive neuromuscular transmission in Kv1.1 null mice: role of potassium channels under the myelin sheath in young nerves. J. Neurosci. 18, 7200-7215.

Zhou, L., et al., 1999. Determinants of excitability at transition zones in Kv1.1-deficient myelinated nerves. J. Neurosci. 19, 5768-5781.

Zhou, L., Chiu, S.Y., 2001. Computer model for action potential propagation through branch point in myelinated nerves. J. Neurophysiol. $85,197-210$.

Zuberi, S.M., et al., 1999. A novel mutation in the human voltage-gated potassium channel gene (Kv1.1) associates with episodic ataxia type 1 and sometimes with partial epilepsy. Brain 122, 817-825. 